\title{
Different Role of Caveolin-1 Gene in the Progression of Gynecological Tumors
}

\author{
Yan Gong ${ }^{1}$, Yuhan Yang ${ }^{2}$, Sufang Tian ${ }^{1 *}$, Honglei Chen ${ }^{1 *}$
}

\begin{abstract}
Caveolin-1 (Cav-1), an integral membrane protein, is a principal component of caveolae and has been reported to play a promoting or inhibiting role in cancer progression. Gynecologic tumor is a group of tumors that affect the tissue and organs of the female reproductive system, especially cervical cancer. Cervical cancer, as one of the most common cancers, severely affects female health in developing countries in particular because of its high morbidity and mortality. This review summarizes some mechanisms of $\mathrm{Cav}-1$ in the development and progression of gynecological tumors. The role of Cav-1 in tumorigenesis, including dysregulation of cell cycle, apoptosis and autophagy, adhesion, invasion, and metastasis, such as the formation of invadopodia and matrix metalloproteinase degradation are presented in detail. In addition, $\mathrm{Cav}$ - 1 modulates autophagy and the formation of invadopodia and target regulated by miRNAs to affect tumor progress. Taken together, we find that, no matter Cav- 1 expression in the tumor or stromal cells, $\mathrm{Cav}-1$ has paradoxical role in different types of gynecological tumors in vivo or in vitro and even in the same tumor from the same organ.
\end{abstract}

Keywords: Caveolin-1- cancer progression- gynecological tumor

Asian Pac J Cancer Prev, 20 (11), 3259-3268

\section{Introduction}

Since the identification of caveolae in epithelial cells of capillary and gall bladder of the mouse in 1950's, it has been found to exist in a wide variety of cell types and tissues, especially in terminally differentiated cells, such as adipocytes, endothelial cells, epithelial cells, fibroblasts, and smooth muscle cells (Scherer et al., 1997). Except for many studies on its location, some progress has also been made regarding the function of caveolae. Caveolae plays an important role in macromolecular transport vesicles, signal transduction, endocytosis, cellular metabolism, cholesterol homeostasis, and tumor progression (Razani et al., 2000b; Galbiati et al., 2001a; Matveev et al., 2001; Parton and Richards, 2003).

Caveolins is an integral membrane protein, whose family includes three members, including caveolin-1/Cav1, caveolin-2/Cav-2 and caveolin-3/Cav-3), is a principal component of caveolae plasma membranes (Glenney and Soppet, 1992). Cav-1, the mostly documented one, has been reported to be down-regulated in different types of human tumor samples, suggesting negative correlation between its expression level and tumor formation and progression, so acting as an tumor suppressor (Bagnoli et al., 2000; Bender et al., 2000). However, Cav-1 has been discovered to be up-regulated in the advanced tumor stage of different kinds of cancers, demonstrating its association with invasion and metastasis (Ho et al.,
2002). The different roles of $\mathrm{Cav}-1$ in cancer may depend on the stage of cancer and its interaction with multiple different signaling molecules in specific tissues and cell types, thus it remains controversial whether $\mathrm{Cav}-1$ acts as an tumor-promoter or a suppressor (Fu et al., 2017).

In order to have a better understanding of the apparent "Cav-1 paradox", a "Cav-1 + partner-X" model has been proposed which considers " $\mathrm{Cav}-1+$ partner-X" as a function integration, but not $\mathrm{Cav}-1$ alone (Burgermeister et al., 2008). For example, $\mathrm{Cav}$ - 1 displays its role as a tumorsuppressor requiring the presence of E-cadherin (Torres et al., 2007). When E-cadherin is still expressed in cancer cells, $\mathrm{Cav}-1$ keeps its anti-proliferative and pro-apoptotic role; whereas, the depletion of E-cadherin resulting in loss of Cav-1 function. Caveolae has been regarded as a message centers, and many signaling molecules taking part in different signaling pathways focus on caveolins areas of the cell surface (Anderson, 1993). Through direct interaction with different signaling molecules by its scaffold areas or regulation of the internalization of signaling molecules to reduce the signals (Burgermeister et al., 2008), the complex of caveolins either promote or suppress tumor occurrence. According to its expression level, Cav-1 is mainly regarded as a tumor suppressor in the early stage of carcinoma since its expression is generally down-regulated in transformed cells; but Cav-1 is suggested to promote cancer progression for its up-regulation in the later stage of some advanced 
carcinomas (Nunez-Wehinger et al., 2014).

Gynecologic tumors are a group of tumors that affect the tissue and organs of the female reproductive system, which often involve the uterus, ovaries, cervix, vulva, vagina, fallopian tubes, or the peritoneum. The most common gynecologic cancer in China is cervical cancer, followed by ovarian cancer and endometrial cancer (Torre et al., 2015). Gestational trophoblastic disease is a gynecologic tumor that may behave aggressively whether being malignant or not. Having a better understanding of the mechanism in tumor progression will greatly contribute to clinical diagnosis, targeted therapy, and prognostic evaluation. Although the role of $\mathrm{Cav}-1$ has been reported in many kinds of tumors, there are a few reports about the role of Cav-1 in gynecological tumors. Therefore, this review summarized some mechanisms and functions of $C a v-1$ in the development and progression of gynecological tumors.

Cav-1 Fuction in Carcinogensis, Invasion, and Metastasis

Tumor formation is a process of interaction among multiple molecules during a long time, dividing into different stages and presenting some specific characteristic in each stage. In the early stage, the principal feature is abnormal proliferation of tumor cells, while invasion and metastasis are remarkable at the later stages. The role of Cav-1 in tumorigenesis, including dysregulation of cell cycle, apoptosis and autophagy, adhesion, and invasion and metastasis, such as the formation of invadopodia and matrix metalloproteinase degradation is presented in detail in the following sections.

\section{Cav-1 inhibits cell cycle}

Cav-1 has been reported to interact with signaling molecules to regulate signaling pathways, such as Wnt, Ras, and p53 and have great effect on inhibiting cell mitogenic and modulating cell cycle. p53 is a key factor in cell cycle arrest and apoptosis promotion through inducing the expression of downstream p21 and p27. Previous studies showed that Cav-1 could mediate cell cycle arrest through $\mathrm{p} 53 / \mathrm{p} 21$ pathway, inducing cells to stay at the G0/G1 stage, reducing cell proliferation and DNA replication rate, because of the increased activity of p53 in NIH $3 \mathrm{~T} 3$ cells transiently expressing Cav1 (Galbiati et al., 2001b). P53 can positively regulate the transcription of $\mathrm{Cav}-1$ gene and protein expression (Razani et al., 2000a). Moreover, the expression of Cav-1 can enhance the activity of p53Cav-1 can also negatively regulate $\mathrm{Wnt} / \beta$-catenin/LEF pathway, that is another kind of mitogenic pathway. Cav- 1 arrests $\beta$-catenin in the caveolin-rich membrane domains and stables the cell adhesion, thus inhibiting $\beta$-catenin to enter nuclear; in addition, it interacts with LEF1 to initial transcription (Galbiati et al., 2000). In agreement, Cav-1 inhibits maximally the cyclinD1 gene promoter, depending on the T-cell factor/lymphoid enhancer factor-1 (TCF/LEF-1) binding site between -81 to -73 in promoter area, which occurs mutation and is abolished the ability to bind TCF protein (Hulit et al., 2000). Furthermore, Cav-1 can inhibit cell cycle through modulating the Ras-p42/p44 MAP kinase pathway. In precise, there is a reciprocal negative regulation relationship between the activation of $\mathrm{p} 42 /$ p44 MAP kinase and Cav-1 expression. In other words, increasing Cav-1 expression can down-regulate p42/p44 MAP kinase activity; on the contrary, enhancing p42/ p44 MAP kinase activity can decrease Cav-1 expression (Engelman et al., 1998). These data demonstrate that Cav-1 can be considered as a tumor-suppressor which is able to limit cell proliferation in the early stage of tumor progression (Figure 1).

\section{Cav-1 promotes cell apoptosis and modulates autophagy}

The PI-3 kinase pathway plays an important role in stimulating cell proliferation, anti-apoptosis, and pro-survival. In the circumstances of desensitization of pro-apoptosis activity resulted from the transfection of Cav-1 antisense vector, the pro-apoptosis activity can restore sensitivity through blocking PI-3 kinase -mediated survival signaling (Liu et al., 2001). Ceramide is reported to have the ability to inhibit PI-3 kinase activity through recruiting PI-3 kinase to Cav-1-enriched membrane domains (Zundel et al., 2000), thus inducing cell apoptosis. Up-regulation of $\mathrm{Cav}-1$ is adequate to limit PI-3 kinase activity and attributes to ceramideinduced apoptosis ; whereas, down-regulation of Cav-1 by antisense repression inversely activates PI-3 kinase and reduces ceramide-induced cell apoptosis. In precise, $\mathrm{Cav}-1$ interacts with epidermal growth factor receptor (EGFR) and $\mathrm{p} 85$, the regulatory subunit of PI-3 kinase, to form a complex, inhibiting PI-3 kinase signaling pathway by inactivating PI-3 kinase, thus enabling ceramide-induced apoptosis. Since EGFR is a component of the complex, the sensitivity of apoptosis may be linked to the inhibition of cell proliferation, because the overexpression of Cav-1 can inhibit the EGF-induced p42/p44 MAP kinase signaling pathway(Engelman et al., 1998), consistent with the report that $\mathrm{Cav}-1$ expression can promote apoptosis activity mediated by inhibition of EGF-induced survival pathways (Razani et al., 2001). Another study showed that Cav-1 plays a role as sensitizing factor or a component of sensitizing complex for cell apoptosis in two cells, namely epithelial (T24) cell and fibroblastic (NIH/3T3) cell (Liu et al., 2001). The above results identified that the character of $\mathrm{Cav}-1$ to inducing pro-apoptosis in agreement with its role acting as an anti-oncogene in the early stage of tumor progression .

It has been proposed that the absence of stromal $\mathrm{Cav}-1$ via autophagy is a negative prognostic factor during tumor progression (Martinez-Outschoorn et al., 2010b; Wu et al., 2018; Qian et al., 2019). The co-culture of human breast cancer cells (MCF7) and immortalized fibroblasts (hTERT-BJ1) or normal human mammary fibroblasts leads to autophagic/lysosomal degradation and down regulation of $\mathrm{Cav}-1$ in fibroblasts, resulting in cancer associated fibroblast (CAFs) phenotype(Martinez-Outschoorn et al., 2010a). To understand tumor metabolism and its invasion through tumor stromal autophagy, a model has been established (Figure 2) (Trimmer et al., 2011). In this model, autophagy in tumor stroma provides fuel for cancer cells, enabling the aggressive growth towards periphery. Mechanically, cancer cells induce the activity of autophagy in CAFs through the loss of $\mathrm{Cav}$-1, promoting oxidative 
stress in adjacent stromal fibroblasts, and subsequently resulting in promoting tumor growth. Cancer cells can also trigger oxidative stress in the microenvironment and activate pro-autophagic promoters in CAFs (Chen and Che, 2014). The autophagy of stromal fibroblasts results in the autophagic loss of Cav-1, and the loss of stromal Cav-1 exacerbates oxidative stress and further promotes autophagy(Zhao et al., 2013). However, contradictory result shows that stromal $\mathrm{Cav}-1$ has a potential to facilitate tumor invasion and metastasis by regulating Rho activity, p190 localization, and phosphorylation (Goetz et al., 2011).

Besides, Cav-1 also modulates autophagy of tumor cells, significantly impacting cancer progression. Cav1 deficiency promotes cell autophagy in breast cancer, and its promoting effect mainly appears at the late stage of autophagy by increasing lysosomal function and autophagosome-lysosome fusion. Furthermore, the elevated autophagy level induced by $\mathrm{Cav}-1$ deficiency can be regarded as a cell survival mechanism under starvation (Shi et al., 2015). Interestingly, Cav-1 can regulate cell apoptosis and autophagy at the same time through 17 $\beta$-estradiol-mediating pathway in human breast cancer cell line, BT474 (Wang et al., 2014). The abundant expression of $\mathrm{Cav}-1$ promotes autophagy and represses cell apoptosis, while deletion of $\mathrm{Cav}-1$ by siRNA represents the adverse result. Except for breast cancer, expression of $\mathrm{Cav}-1$ contributes to tumor growth and metastasis through inhibiting autophagy in hepatocellular carcinoma (Liu et al., 2016). In general, the loss of stromal Cav-1 through autophagy is a negative prognostic factor during tumor progression, while the autophagy of tumor cells modulated by $\mathrm{Cav}-1$ has different function in cancer progression.

\section{Cav-1 enhances cell adhesion}

Cadherin is an important adhesion related molecule, which is supposed to be an invasion-suppression factor acting as an anti-oncogene. $\mathrm{Cav}-1$ has been validated to maintain epithelial and endothelial barriers as a participating protein targeted to adherens and tight junction (Miotti et al., 2005; Schwarz et al., 2007). Thus, the interaction between $C a v-1$ and cadherin may greatly influence cell adhesion and tumor cell metastasis (Figure $3)$. $\beta$-catenin is a component of adhesive complexes, which stables adhesion between adjacent cells. However, when it is separated from E-cadherin and translocated to cell nucleus, $\beta$-catenin initials gene transcription. Therefore, both expression and distribution of $\beta$-catenin are critical in modulating cell-cell adhesion and gene transcription. Up-regulation of Cav-1 expression has been reported to recruit $\beta$-catenin to caveolae membranes to stable adhesion complexes and reinforce cell-cell adhesion; meanwhile, prevent the entrance of $\beta$-catenin to cell nucleus and inhibit $\beta$-catenin/LEF-1 signaling pathway (Galbiati et al., 2000). EGF-induced down-regulation of $\mathrm{Cav}-1$ plays a vital role in the complexe changes leading to metastasis (Lu et al., 2003). It is found that chronic EGF treatment can down-regulate $\mathrm{Cav}-1$ and E-cadherin expressions, inducing $\beta$-catenin separation from E-cadherin and translocation to nucleus and enhancing $\beta$-cateninTCF/LEF-1 signaling and transcriptional activity, subsequently resulting in disruption of cell-cell adhesion and epithelial-to-mesenchymal transition (EMT). These data suggest that $\mathrm{Cav}-1$ expression promotes cell adhesion, thus can suppress the tumor progression.

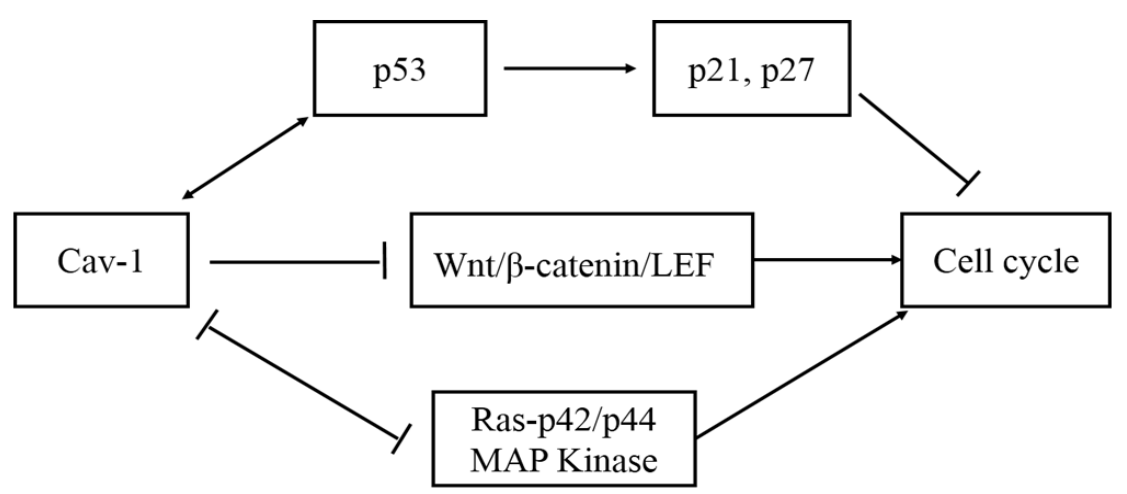

Figure. 1 The Mechanism of Cav-1 Inhabits Cell Cycle. There are three pathways: enhancing the activity of p53 to promote downstream expression of $\mathrm{p} 21$ and $\mathrm{p} 27$; inhibiting $\mathrm{Wnt} / \beta$-catenin/LEF pathway; negatively regulating Ras-p42/p44 MAP kinase pathway.

Table 1. The mi-RNA and Targeting Caveolins in Cancers

\begin{tabular}{lccc}
\hline mi-RNA & Targeting caveolin & Types of cancer & References \\
\hline miR-124-3p & Cav-1 & clear cell renal cell carcinoma & (Butz et al., 2015) \\
miR-133a & Cav-1 & head and neck squamous cell carcinoma & (Nohata et al., 2011) \\
miR107 & Cav-1 & pancreatic ductal adenocarcinoma & (Xiong et al., 2017) \\
miR-203 & Cav-1 & pancreatic cancer & (Miao et al., 2014) \\
miR-199a-3p & Cav-2 & breast cancer & (Shatseva et al., 2011) \\
miR-218 & $C a v-2$ & renal cell carcinoma & (Yamasaki et al., 2013) \\
\hline
\end{tabular}


Table 2. The Promoting Role of Cav-1 in Gynecological Tumor

\begin{tabular}{|c|c|c|c|}
\hline Types of tumor & The promoting role of $\mathrm{Cav}-1$ expression & Study type & References \\
\hline Cervical cancer & Enhancing cell proliferation & In vivo & (Sun et al., 2012) \\
\hline Ovarian cancer & $\begin{array}{l}\text { Often expressed in advanced-stage ovarian carcinoma, promoting } \\
\text { chemotherapy resistance, inhibiting cell apoptosis }\end{array}$ & $\begin{array}{l}\text { In vivo } \\
\text { In vitro }\end{array}$ & $\begin{array}{l}\text { (Davidson et al., 2001; } \\
\text { Zou et al., 2015) }\end{array}$ \\
\hline Endometrial cancer & Increasing cells anchorage-independent growth, invasion and migration & $\begin{array}{l}\text { In vivo } \\
\text { In vitro }\end{array}$ & (Diaz-Valdivia et al., 2015) \\
\hline Choriocarcinoma & Promoting proliferation and invasion & In vitro & (Liu et al., 2008) \\
\hline Uterine leiomyosarcomas & $\begin{array}{l}\text { Perivascular } C a v-1 \text { expression in leiomyosarcomas was stronger than } \\
\text { that of leiomyomas; } C a v-1 \text { was observed in most cells in leiomyomas } \\
\text { and in only few cells in normal smooth muscle cells }\end{array}$ & In vivo & $\begin{array}{l}\text { (Sendemir et al., 2008; } \\
\text { Ayaz et al., 2016) }\end{array}$ \\
\hline
\end{tabular}

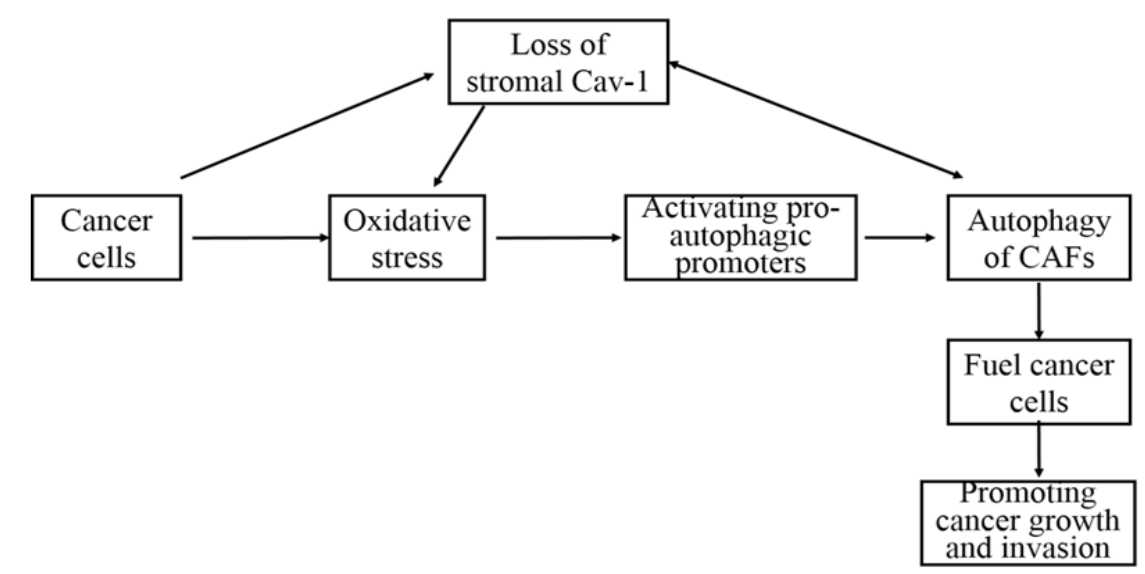

Figure 2. The Association between Stromal Cav-1, Oxidative Stress and Cancer Progression in Tumor Microenvironment

The main feature of normal cell growth is anchorage-dependent proliferation, it going to anchorage-independent induced apoptosis upon cell detachment. When cell detaches, the signal subsequently triggers Cav-1 endocytosis accompany by down-regulating integrin signaling and inducing apoptosis (del Pozo et al., 2005; Del Pozo and Schwartz, 2007). Signaling pathways, such as Erk, PI-3 kinase and Rac need integrin-mediated cell adhesion. On the other hand, the components of these signaling pathways locate to lipid rafts, the same place caveolins localization. According to caveolae signaling hypothesis, various inactive signaling molecules are located in caveolar, laying the basis of subsequent molecules activation and cross-talk between different signaling pathways (Lisanti et al., 1994). Integrin-mediated adhesion can modulate lipid rafts trafficking such that upon cell detachment, the phosphorylation of $\mathrm{Cav}-1$ and its location change from focal adhesions to caveolae induce lipid rafts internalization and clearance, resulting in inhibition of PI-3 kinase, Erk, and Rac, thus suppressing tumor growth (del Pozo et al., 2005). The phenomenon that Cav-1 promotes cells apoptosis or down-regulates downstream signaling pathways following cells detachment is beneficial for the prevention of tumor formation.

\section{Cav-1 and invadopodia}

Some documents demonstrate that $\mathrm{Cav}-1$ represses the formation of invadopodia. A relationship between Cav-1, cholesterol and invasion in cancer progression indicates that lower cholesterol is beneficial to inhibit the formation of invadopodia (Caldieri et al., 2009). The appropriate levels of cholesterol in plasma membrane decide the formation, structural integrity, and function of

Table 3. The Inhibiting Role of Cav-1 in Gynecological Tumor

\begin{tabular}{|c|c|c|c|}
\hline Types of tumor & The inhibiting role of $C a v-1$ expression & $\begin{array}{l}\text { Study } \\
\text { type }\end{array}$ & References \\
\hline Cervical cancer & $\begin{array}{l}\text { Inactivation through gene mutation or promoter methylation in cancer } \\
\text { repression, inhibiting proliferation and cell viability, and inducing } \\
\text { apoptosis }\end{array}$ & $\begin{array}{l}\text { In vivo } \\
\text { In vitro }\end{array}$ & $\begin{array}{l}\text { (Chan et al., 2003; Zhang et al., } \\
\text { 2017a; Zhang et al., 2017b) }\end{array}$ \\
\hline Endometrial cancer & $\begin{array}{l}\text { Down-regulation through Human Genome GeneChip in type } 1 \\
\text { endometrial cancer }\end{array}$ & In vivo & (Saghir et al., 2010) \\
\hline Ovarian tumor & $\begin{array}{l}\text { Stromal } C a v-1 \text { regarded as a marker to distinguish benign and } \\
\text { borderline tumors, } C a v-1 \text { forming a complex with E-cadherin to } \\
\text { stabilize cell-cell adhesion, anti-proliferation, pro-apoptosis and } \\
\text { inhibiting invasion, facilitating the uptake of cisplatin nanocapsules }\end{array}$ & $\begin{array}{l}\text { In vivo } \\
\text { In vitro }\end{array}$ & $\begin{array}{l}\text { (Davidson et al., 2001; Wiechen et } \\
\text { al., 2001; Miotti et al., 2005; Syed } \\
\text { et al., 2005; Hamelers et al., 2009; } \\
\text { Sayhan et al., 2015) }\end{array}$ \\
\hline Uterine leiomyomas & $\begin{array}{l}\text { Down-regulation in human uterine leiomyomas compared to normal } \\
\text { myometrial tissues }\end{array}$ & $\begin{array}{l}\text { In vivo } \\
\text { In vitro }\end{array}$ & (Zhou et al., 2014) \\
\hline
\end{tabular}




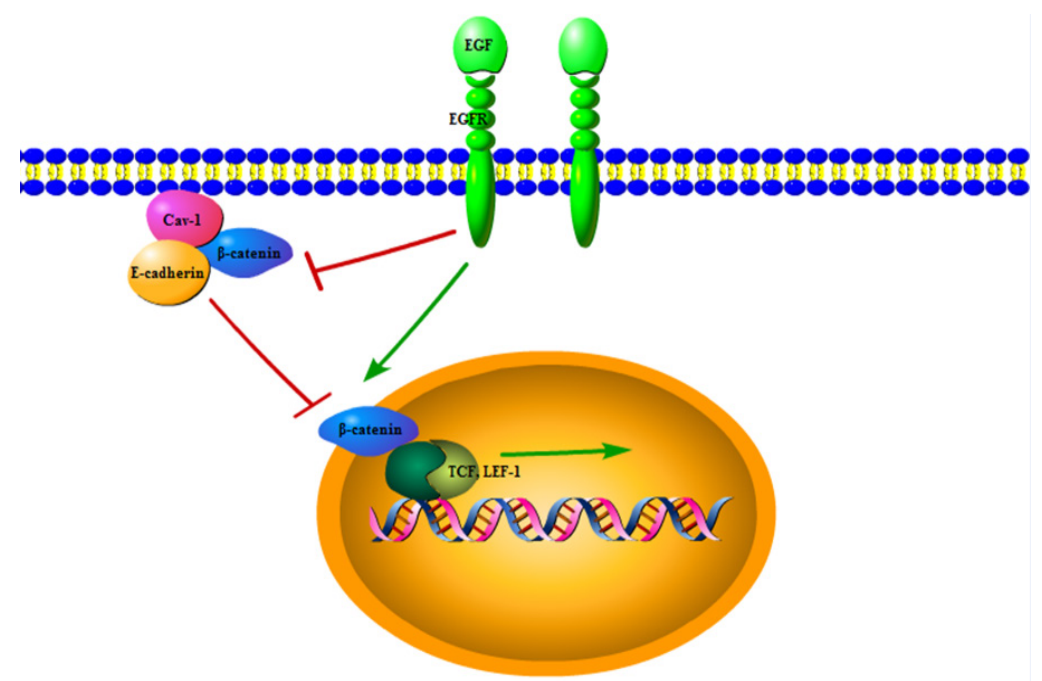

Figure 3. Expression of $C a v-1$ can Enhance Cell Adhesion. Up-regulation of $C a v-1$ expression can recruit $\beta$-catenin to caveolae membranes to stable adhesion complexes, thereby, reinforcing cell-cell adhesion; at the same time, prevent the entrance of $\beta$-catenin to cell nucleus and inhibit $\beta$-catenin/LEF-1 signal. EGF binding with EGFR can down-regulate $C a v-1$ and $E$-cadherin expression, inducing $\beta$-catenin separating from E-cadherin to nucleus and enhancing $\beta$-catenin-TCF/LEF-1 signal.

invadopodia. On the other hand, $\mathrm{Cav}-1$ is a key regulator for invadopodia formation acting through modulating cholesterol levels at the plasma membrane. The phosphorylation of $\mathrm{Cav}-1$ tyrosine impedes the formation of invadopodia by mediating the internalization of cholesterol enriched membrane microdomains (CEMM) from the plasma membrane upon cell detachment. Similarly, metastatic mammary adenocarcinoma cells (MTLn3) which represents a rapid lamellipod extension and cell migration induced by EGF fails to express endogenous $\mathrm{Cav}-1$ at detectable levels (Zhang et al., 2000). Re-expression of Cav-1 in MTLn 3 can inhibit the EGF-induced lamellipod biogenesis and cell migration, prevent the anchorage-independent growth, and suppress the invasion and metastasis of tumor cells.

However, contradictory views in this regard represent a promoting role in invadopodia formation. Cell polarization and directional movement can be prevented by $\mathrm{Cav}-1$ polarity loss because of the targeted down-regulation of Cav-1 (Beardsley et al., 2005). In the process of cells migration, $C a v-1$ is found to be in the posterior of moving cells. In addition, $C a v-1$ and lipid rafts are required for the formation and activity of invadopodia and extracellular matrix (ECM) degradation in human breast cancer cells (Yamaguchi et al., 2009). Cav-1 and membrane type 1 matrix metalloproteinase (MT1-MMP) co-localize and co-express in invadopodia, resulting in ECM degradation and tumor invasion and migration (Ebisawa et al., 2015; Yang et al., 2016). The activation of Cav-1 and PI3K/ $\mathrm{Akt} / \mathrm{mTOR}$ signaling under low shear stress increases the MT1-MMP expression, invadopodia formation, and ECM degradation and promotes breast cancer cell motility and metastasis(Yang et al., 2016). These results seem to be contradictory regarding the proposed function for $\mathrm{Cav}-1$, a tumor suppressor, since the expression of $\mathrm{Cav}-1$ is a promoting factor in invadopodia formation and migration ability.

\section{Cav-1 inhibits ECM degradation}

The degradation of ECM contributes to tumor cells invading into blood and lymphatic vessels and promotes tumor invasion and metastasis. Cav-1 is suggested

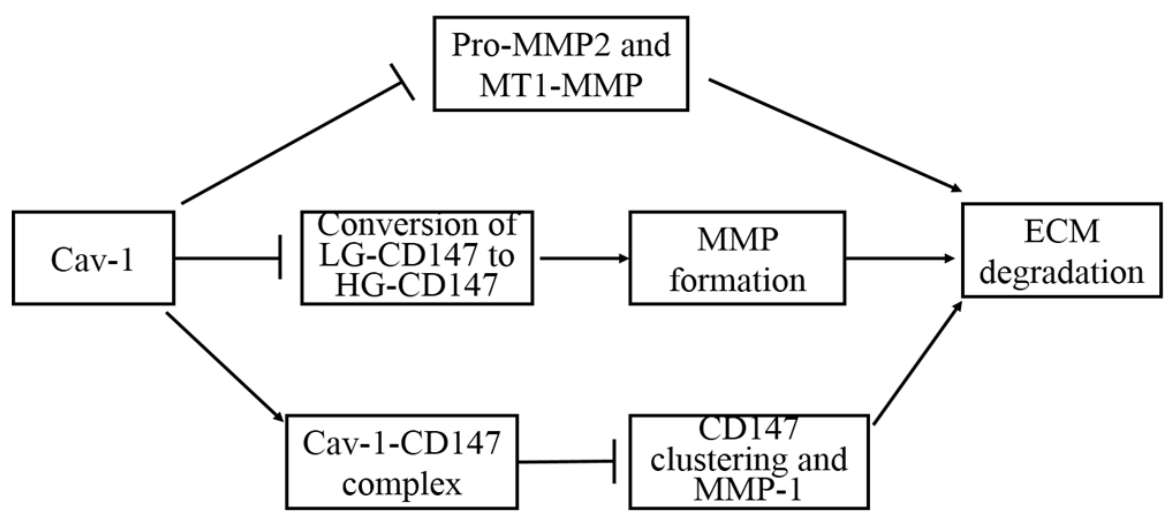

Figure 4. Cav-1 Inhibits ECM Degradation Through Repressing the Formation and Activity of MMP. Inhibiting the activity of Pro-MMP2 and MT1-MMP through MT1-MMP internalization; restraining the conversion of low glycosylation $C D 147$ ( $L G$-CD147) to high glycosylation (HG-CD147) to decrease MMP formation; Forming Cav-1/ $C D-147$ complex to diminish CD147 clustering in the cell surface and CD147-dependent MMP-1 activity. 
to attenuate proMMP-2 activity and down-regulate MT1-MMP activity by promoting internalization of MT1-MMP from the cell surface (Kim and Chung, 2008), consequently resulting in ECM cleavage reduction and cell migration inhibition. MT1-MMP can degrade ECM and activate proMMP-2, thus increasing the degradation of ECM, promoting ECM remodeling, and contributing to cancer cell invasion and metastasis. In murine mammary glands, loss of Cav-1 can increase the expression of ECM and $\alpha$-SMA protein and change ductal architecture, which may lead to tumor initiation(Thompson et al., 2017). In addition, $C a v-1$ has been reported to form a complex with CD147, diminishing CD147 clustering in the cell surface and CD147-dependent MMP-1 activity (Tang and Hemler, 2004). Also, Cav-1 is demonstrated to restrain the conversion of low glycosylation CD147 to high glycosylation (Tang et al., 2004), inhibiting MMP induction and ECM degradation and acting as tumor suppressor because high glycosylation CD147 can stimulate the induction of MMP (Figure 4). Through modulating MMP activity and formation and reducing MMP-induced ECM degradation, Cav-1negatively regulates metastasis progression as an anti-oncogene.

\section{Cav-1, Cav-2, and miRNAs}

Many documents have demonstrated that caveolins are the target of miRNAs in regulating tumor cells proliferation, survival, invasion, and migration. MiR-124-3p, -200c-3p, and $-30 a-5 p$ are the most influential miRNAs, which are down-regulated in clear cell renal cell carcinoma (ccRCC) through constructing miRNA-target interaction network (Butz et al., 2015). Mechanically, miR-124-3p has a key role in repressing tumor progression by targeting $\mathrm{Cav}-1$ and Flotillin 1 (FLOT1). The higher expression of $\mathrm{Cav}-1$ and FLOT1 is associated with lower miR-124-3p expression and poor survival of ccRCC. Restoration of the expression of these miRNAs can reduce the proliferation, invasion, and migration of tumor cells. Thus, re-expression of these miRNAs can be considered as a potential therapeutic strategy in ccRCC. These results consist with those of a previous research revealing that $m i R-133 a$ regulates the expression of $C a v-1$ which mediates tumor cell invasion and migration in head and neck squamous cell carcinoma (SCC) (Nohata et al., 2011). Here, mi-RNAs inhibit cancer progression, while caveolins promote it; moreover, the expression level of caveolins is negatively regulated by mi-RNAs. Conversely, miR-107 is highly expressed in the pancreatic ductal adenocarcinoma tissues and cells, which is associated with poor prognosis. Low expression of miR-107 due to the inhibitor transfection inhibits cell migration and invasion by up-regulating $C a v-1$ and PTEN (Xiong et al., 2017).

MiR-203 is suggested to repress tumor invasion and migration through Cav-1 in pancreatic cancer cells (Panc-1 cells) (Miao et al., 2014). However, miR-203 positively regulates the expression of $\mathrm{Cav}-1$ and plays a synergistic effect in inhibiting tumor invasion and migration in Panc-1 cells. Besides, Cav-1 is essential for miR-203-dependent tumor cells invasion and migration inhabitation. Transfection of miR-203 alone in Cav-1 knockdown Panc-1 cells fails to suppress cell invasion and migration, but significantly elevates cell migration.

Except for Cav-1, Cav-2 is also reported as a target of mi-RNAs in MDA-MB-231 and MT-1 breast cancer cell lines (Shatseva et al., 2011). MiR-199a-3p promotes cell proliferation and survival in MDA-MB-231, MT-1 breast cancer cells, and YPEN-1 endothelial cells lines by inhibiting endogenous and exogenous Cav-2. Overexpression of Cav-2 completely offsets the effect of MiR-199a-3p in cell proliferation and survival. In a previous study, MiR-199a-3p contributed to breast cancer progression; whereas, $\mathrm{Cav}-2$ repressed cancer progression. In contrast, $C a v-2$, targeting negatively regulated by miR218 , was reported to promote cell invasion and migration in renal cell carcinoma through dysregulation of focal adhesion (Yamasaki et al., 2013).

Although the promoting or inhibiting role of miRNAs and caveolins in different types of cancer is reported, the targeting role of caveolins regulated by miRNAs in cancer progression has been identified clearly.

\section{CAV1 Expression in Gynecological Tumor}

The expression of Cav-1 is distinct in different cell types and tissues and also in different stages of tumor(Fu et al., 2017), so the levels of Cav-1 mRNA and protein are valuable to be discussed in gynecological tumor. The role of Cav-1 in gynecological tumor is also paradoxical acting a promoting or inhibiting role in tumor progression (Table 2, Table 3). or not a potential biomarker of uterine malignant mesenchymal tumors (Hayashi et al., 2015). On the other hand, there are very few reports on the role of $C a v-1$ expression in vaginal cancer, vulvar cancer, and fallopian tube cancer. In the following, paradoxical roles of $C a v-1$ are explained in more details.

\section{The promoting role of $\mathrm{Cav}-1$}

Some studies have suggested that Cav-1 promotes tumor progression. In other words, higher expression of $C a v-1$ contributes to the incidence and development of gynecological tumor. In cervical SCC, expression of $C a v-1$ in tumor cells possibly contributes to cancer progression through enhancing cell proliferation, but little connection was reported between stromal tissues and $\mathrm{Cav}$ 1 expression (Sun et al., 2012). Through gene ontology analysis, $\mathrm{Cav}-1$ was revealed as one of key node genes for cervical SCC invasion and metastasis (Cheng et al., 2015). In ovarian cancer, it was found that the expression of Cav-1 was frequently presented in advanced ovarian carcinomas and more expressed at cell membrane with metastasis and poor-survival tumors (Davidson et al., 2001). Besides, it was revealed that Cav-1 promoted chemotherapy resistance in ovarian cancer through

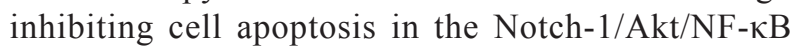
pathway (Zou et al., 2015). In a nutshell, it can be concluded that cisplatin-induced apoptotic ratio is higher in common ovarian cancer compared with ovarian cancer cells with cisplatin-resistance; moreover, it seems that down-regulation of Cav-1 expression by knockdown of Cav-1 can increase cisplatin-induced cell apoptosis.

Cancer cells metastasis significantly promotes cancer progression. Up-regulated expression of $\mathrm{Cav}-1$ induced by the activation of tumor promoter 12-O-tetradecanoyl- 
phorbol-13-acetate (4 $\beta$-TPA) is associated with enhanced malignancy behavior of endometrial cancer cells through increasing cells anchorage-independent growth, invasion, and migration (Diaz-Valdivia et al., 2015). Additionally, it was reported that $\mathrm{Cav}-1$ could promote the invasion ability of choriocarcinoma cells, and the repressive expression of $C a v-1$ by si-RNA could limit the proliferation and invasion of choriocarcinoma cells (Liu et al., 2008). Perivascular Cav-1 expression is considered as a marker for distinguishing benign from malignant uterine smooth muscle tumors given that perivascular Cav-1 is more expressed in leiomyosarcomas compared with uncertain malignant potentials and leiomyomas (Ayaz et al., 2016). The immunoreactivity for Cav-1 is observed in most cells of leiomyomas. Only few cells in normal myometrial tissues, and mostly co-localized with sex hormone-binding globulin (SHBG) and oxytocin (OT) in leiomyoma samples, which may be linked to growth of leiomyomas (Sendemir et al., 2008).

\section{The inhibiting role of Cav-1}

Some studies indicate that the normal and elevated expression of $C a v-1$ can significantly inhibit tumor cells proliferation, invasion, and metastasis, thus it can be concluded that the function loss and down-regulation of Cav-1 is a meaningful marker in cancer progression. Sclareol is reported to inhibit cell proliferation and sensitize cells to bortezomib through increasing the expression of Cav-1 and down-regulating superoxide dismutase1 (SOD1) protein level in cervical cancer cells (Zhang et al., 2017b). Similarly, increased expression of Cav-1 and mitochondrial carrier homolog 2 (MTCH2) after dihydroartemisinin treatment activate $\mathrm{p} 53$, leading to HeLa cell viability inhibition and apoptosis induction (Zhang et al., 2017a). Cav-1 is critical in the uptake and high cytotoxicity of Cisplatin Nanocapsules through caveolae-mediated endocytosis in ovarian carcinoma cells (Hamelers et al., 2009). It is suggested that $C a v-1$ is a critical molecule in the treatment of anti-cancer drug. Cav-1 can lose its function in cancer repression following inactivation through gene mutation or promoter methylation. Cav-1 loses its suppressing role in the development of cervical cancer, including 54 SCCs and 18 adenocarcinomas, by methylation of Cav-1 promoter or other unknown mechanisms (Chan et al., 2003). Having used a new version of Affymetrix Human Genome GeneChip 1.0 ST array, Cav-1 gene is down-regulated in type I endometrial carcinoma(Saghir et al., 2010). Cav-1 expression in stromal and perivascular is almost presented in benign ovarian serous tumors, while the Cav-1 expression is lost in the majority of malignant and borderline tumors, indicating that the expression levels of stroma Cav-1 can act as a marker to distinguish the benign and borderline ovarian tumors (Sayhan et al., 2015). A previous study indicated that $\mathrm{Cav}-1$ displayed a tumor-suppressor role, which requiring the presence of E-cadherin (Torres et al., 2007). Interestingly, the neo-expression of E-cadherin in previous E-cadherin down-regulated ovarian carcinoma did not inhibit the release and migration of cells from the primary tumor because the expression of $\mathrm{Cav}-1$ was down-regulated even though E-cadherin level was adequate (Miotti et al., 2005). The simultaneous expression of E-cadherin and $\mathrm{Cav}-1$ is essential to stabilize cell-cell adhesion and play a tumor suppressor role. In addition, hepatocyte growth factor (HGF) down-regulates E-cadherin, betacatenin, and caveolin-1, disassembly of cell-cell contacts, and invasion and migration enhancement in human ovarian cancer cells ( $\mathrm{Hu}$ et al., 2010). It is suggested that the expression of $\mathrm{Cav}-1$ can indirectly promote cellcell adhesion by inhibiting src-related kinases. Thus, down-regulation of $\mathrm{Cav}-1$ together with increase of src kinase activity might greatly contribute to the migration of ovarian tumor cells by destructing cell adhesion (Prinetti et al., 2011). Another study indicated that Cav-1 expression was presented at normal ovarian epithelial cells and benign tumor cells, but was absent in serous ovarian carcinoma (Wiechen et al., 2001). Moreover, loss of Cav-1 can induce disease-associated mutant BRCA1 proteins in serous epithelial ovarian cancer cells (Xu et al., 2014). Overexpression of $\mathrm{Cav}-1$ can promote apoptosis of ovarian cancer cells after galectin-3 and paclitaxel treatment (Cai et al., 2016). The re-expression of Cav-1 in OVCAR-3, an ovarian carcinoma cell line, prevents tumor cell survival in vitro. Consistently, Cav-1 is reduced in ovarian cancer cell lines compared to human ovarian surface epithelial (HOSE) cell lines and upregulated following progesterone $(\mathrm{P} 4)$ treatment. Ectopic expression of $\mathrm{Cav}-1$ leads to growth inhibition, activation of caspase-3, reduction of colony formation, and cell invasiveness in ovarian cancer (Syed et al., 2005). The above results demonstrate that $\mathrm{Cav}-1$ is down-regulated in human ovarian carcinoma and acts as a candidate of tumor suppressor gene (Wiechen et al., 2001). In uterine leiomyomas, $C a v-1$ is down-regulated compared to normal myometrial tissues (Zhou et al., 2014), suggesting that low expression levels of $\mathrm{Cav}-1$ may promote the pathological process. Therefore, the up-regulation of Cav- 1 expression by gene therapy may significantly inhibit some gynecological tumors progression.

\section{Perspectives and Conclusion}

$C a v-1$ has been reported to play a promoting or inhibiting role in cancer progression in many types of tumors, but there are only a few published studies on the role of Cav-1 in gynecological tumor. This review summarized some mechanisms of Cav-1 in cancer progression to profoundly reveal the association between Cav-1 expression and gynecological tumors. Regardless of Cav-1 expression in the tumor or stromal cells, we found that $\mathrm{Cav}-1$ also had paradoxical role in different types of gynecological tumors, both in vivo and in vitro and in the same tumor from the same organ. However, there is no document investigating the intrinsic mechanism of Cav-1. Herein, further investigations are needed to study the role of $\mathrm{Cav}^{-1}$ in the carcinogenesis of gynecological tumors.

\section{Acknowledgments}

This work was supported by grants from the Natural Science Foundation of Hubei Province (No:ZRY2015001716). We thank Dr. Pronnaphat 
Phiphatwatchara providing English polish support.

\section{References}

Anderson RG (1993). Caveolae: where incoming and outgoing messengers meet. Proc Natl Acad Sci U S A, 90, 10909-13.

Ayaz D, Diniz G, Kahraman DS, et al (2016). The evaluation of the caveolin-1 and AT-rich interactive domain 1 alpha expressions in uterine smooth muscle tumors. Indian $J$ Pathol Microbiol, 59, 301-4.

Bagnoli M, Tomassetti A, Figini M, et al (2000). Downmodulation of caveolin-1 expression in human ovarian carcinoma is directly related to alpha-folate receptor overexpression. Oncogene, 19, 4754-63.

Beardsley A, Fang K, Mertz H, et al (2005). Loss of caveolin-1 polarity impedes endothelial cell polarization and directional movement. $J$ Biol Chem, 280, 3541-7.

Bender FC, Reymond MA, Bron C, et al (2000). Caveolin-1 levels are down-regulated in human colon tumors, and ectopic expression of caveolin-1 in colon carcinoma cell lines reduces cell tumorigenicity. Cancer Res, 60, 5870-8.

Burgermeister E, Liscovitch M, Rocken C, et al (2008). Caveats of caveolin-1 in cancer progression. Cancer Lett, 268, 187-201.

Butz H, Szabo PM, Khella HW, et al (2015). miRNA-target network reveals miR-124as a key miRNA contributing to clear cell renal cell carcinoma aggressive behaviour by targeting CAV1 and FLOT1. Oncotarget, 6, 12543-57.

Cai G, Ma X, Chen B, et al (2016). Galectin-3 induces ovarian cancer cell survival and chemoresistance via TLR4 signaling activation. Tumour Biol, 37, 11883-91.

Caldieri G, Giacchetti G, Beznoussenko G, et al (2009). Invadopodia biogenesis is regulated by caveolin-mediated modulation of membrane cholesterol levels. $J$ Cell Mol Med, 13, 1728-40.

Chan TF, Su TH, Yeh KT, et al (2003). Mutational, epigenetic and expressional analyses of caveolin-1 gene in cervical cancers. Int J Oncol, 23, 599-604.

Chen D, Che G (2014). Value of caveolin-1 in cancer progression and prognosis: Emphasis on cancer-associated fibroblasts, human cancer cells and mechanism of caveolin-1 expression (Review). Oncol Lett, 8, 1409-21.

Cheng Y, Ma D, Zhang Y, et al (2015). Cervical squamous cancer mRNA profiles reveal the key genes of metastasis and invasion. Eur J Gynaecol Oncol, 36, 309-17.

Davidson B, Nesland JM, Goldberg I, et al (2001). Caveolin-1 expression in advanced-stage ovarian carcinoma--a clinicopathologic study. Gynecol Oncol, 81, 166-71.

del Pozo MA, Balasubramanian N, Alderson NB, et al (2005). Phospho-caveolin-1 mediates integrin-regulated membrane domain internalization. Nat Cell Biol, 7, 901-8.

Del Pozo MA, Schwartz MA (2007). Rac, membrane heterogeneity, caveolin and regulation of growth by integrins. Trends Cell Biol, 17, 246-50.

Diaz-Valdivia N, Bravo D, Huerta H, et al (2015). Enhanced caveolin-1 expression increases migration, EGFR growth and invasion of endometrial adenocarcinoma cells. $B M C$ Cancer, 15, 463.

Ebisawa M, Iwano H, Nishikawa M, et al (2015). Significance of caveolin- 1 and matrix metalloproteinase 14 gene expression in canine mammary tumours. Vet $J, 206,191-6$.

Engelman JA, Chu C, Lin A, et al (1998). Caveolin-mediated regulation of signaling along the p42/44 MAP kinase cascade in vivo. A role for the caveolin-scaffolding domain. FEBS Lett, 428, 205-11.

Fu P, Chen F, Pan Q, et al (2017). The different functions and clinical significances of caveolin-1 in human adenocarcinoma and squamous cell carcinoma. Onco Targets Ther, 10, 819-35. Galbiati F, Razani B, Lisanti MP (2001a). Emerging themes in lipid rafts and caveolae. Cell, 106, 403-11.

Galbiati F, Volonte D, Brown AM, et al (2000). Caveolin-1 expression inhibits Wnt/beta-catenin/Lef-1 signaling by recruiting beta-catenin to caveolae membrane domains. $J$ Biol Chem, 275, 23368-77.

Galbiati F, Volonte D, Liu J, et al (2001b). Caveolin-1 expression negatively regulates cell cycle progression by inducing $\mathrm{G}(0) / \mathrm{G}(1)$ arrest via a p53/p21(WAF1/Cip1)-dependent mechanism. Mol Biol Cell, 12, 2229-44.

Glenney JR Jr, Soppet D (1992). Sequence and expression of caveolin, a protein component of caveolae plasma membrane domains phosphorylated on tyrosine in Rous sarcoma virus-transformed fibroblasts. Proc Natl Acad Sci U S A, 89, 10517-21.

Goetz JG, Minguet S, Navarro-Lerida I, et al (2011). Biomechanical remodeling of the microenvironment by stromal caveolin-1 favors tumor invasion and metastasis. Cell, 146, 148-63.

Hamelers IH, Staffhorst RW, Voortman J, et al (2009). High cytotoxicity of cisplatin nanocapsules in ovarian carcinoma cells depends on uptake by caveolae-mediated endocytosis. Clin Cancer Res, 15, 1259-68.

Hayashi T, Ichimura T, Yaegashi N, et al (2015). Expression of CAVEOLIN 1 in uterine mesenchymal tumors: No relationship between malignancy and CAVEOLIN 1 expression. Biochem Biophys Res Commun, 463, 982-7.

Ho CC, Huang PH, Huang HY, et al (2002). Up-regulated caveolin-1 accentuates the metastasis capability of lung adenocarcinoma by inducing filopodia formation. $A m J$ Pathol, 161, 1647-56.

Hu J, Shao S, Song Y, et al (2010). Hepatocyte growth factor induces invasion and migration of ovarian cancer cells by decreasing the expression of E-cadherin, beta-catenin, and caveolin-1. Anat Rec (Hoboken), 293, 1134-9.

Hulit J, Bash T, Fu M, et al (2000). The cyclin D1 gene is transcriptionally repressed by caveolin-1. J Biol Chem, 275, 21203-9.

Kim HN, Chung HS (2008). Caveolin-1 inhibits membrane-type 1 matrix metalloproteinase activity. BMB Rep, 41, 858-62.

Lisanti MP, Scherer PE, Tang Z, et al (1994). Caveolae, caveolin and caveolin-rich membrane domains: a signalling hypothesis. Trends Cell Biol, 4, 231-5.

Liu HN, Cai JT, Lin QH, et al (2008). Expression of caveolin-1 and the invasion of choriocarcinoma. Zhong Nan Da Xue Xue Bao Yi Xue Ban, 33, 331-7.

Liu J, Lee P, Galbiati F, et al (2001). Caveolin-1 expression sensitizes fibroblastic and epithelial cells to apoptotic stimulation. Am J Physiol Cell Physiol, 280, 823-35.

Liu WR, Jin L, Tian MX, et al (2016). Caveolin-1 promotes tumor growth and metastasis via autophagy inhibition in hepatocellular carcinoma. Clin Res Hepatol Gastroenterol, 40, 169-78.

$\mathrm{Lu}$ Z, Ghosh S, Wang Z, et al (2003). Downregulation of caveolin-1 function by EGF leads to the loss of E-cadherin, increased transcriptional activity of $\beta$-catenin, and enhanced tumor cell invasion. Cancer Cell, 4, 499-515.

Martinez-Outschoorn UE, Pavlides S, Whitaker-Menezes D, et al (2010a). Tumor cells induce the cancer associated fibroblast phenotype via caveolin-1 degradation: implications for breast cancer and DCIS therapy with autophagy inhibitors. Cell Cycle, 9, 2423-33.

Martinez-Outschoorn UE, Whitaker-Menezes D, Pavlides S, et al (2010b). The autophagic tumor stroma model of cancer or "battery-operated tumor growth": A simple solution to the autophagy paradox. Cell Cycle, 9, 4297-306. 
Matveev S, Li X, Everson W, et al (2001). The role of caveolae and caveolin in vesicle-dependent and vesicle-independent trafficking. Adv Drug Deliv Rev, 49, 237-50.

Miao L, Xiong X, Lin Y, et al (2014). miR-203 inhibits tumor cell migration and invasion via caveolin-1 in pancreatic cancer cells. Oncol Lett, 7, 658-62.

Miotti S, Tomassetti A, Facetti I, et al (2005). Simultaneous expression of caveolin-1 and E-cadherin in ovarian carcinoma cells stabilizes adherens junctions through inhibition of src-related kinases. Am J Pathol, 167, 1411-27.

Nohata N, Hanazawa T, Kikkawa N, et al (2011). Caveolin-1 mediates tumor cell migration and invasion and its regulation by miR-133a in head and neck squamous cell carcinoma. Int J Oncol, 38, 209-17.

Nunez-Wehinger S, Ortiz RJ, Diaz N, et al (2014). Caveolin-1 in cell migration and metastasis. Curr Mol Med, 14, 255-74.

Parton RG, Richards AA (2003). Lipid rafts and caveolae as portals for endocytosis: new insights and common mechanisms. Traffic, 4, 724-38.

Prinetti A, Cao T, Illuzzi G, et al (2011). A glycosphingolipid/ caveolin-1 signaling complex inhibits motility of human ovarian carcinoma cells. J Biol Chem, 286, 40900-10.

Qian XL, Pan YH, Huang QY, et al (2019). Caveolin-1: a multifaceted driver of breast cancer progression and its application in clinical treatment. Onco Targets Ther, 12, 1539-52.

Razani B, Altschuler Y, Zhu L, et al (2000a). Caveolin-1 expression is down-regulated in cells transformed by the human papilloma virus in a p53-dependent manner. Replacement of caveolin-1 expression suppresses HPV-mediated cell transformation. Biochemistry, 39, 13916-24.

Razani B, Schlegel A, Lisanti MP (2000b). Caveolin proteins in signaling, oncogenic transformation and muscular dystrophy. J Cell Sci, 113, 2103-9.

Razani B, Schlegel A, Liu J, et al (2001). Caveolin-1, a putative tumour suppressor gene. Biochem Soc Trans, 29, 494-9.

Saghir FS, Rose IM, Dali AZ, et al (2010). Gene expression profiling and cancer-related pathways in type I endometrial carcinoma. Int J Gynecol Cancer, 20, 724-31.

Sayhan S, Diniz G, Karadeniz T, et al (2015). Expression of caveolin-1 in peritumoral stroma is associated with histological grade in ovarian serous tumors. Ginekol Pol, 86, 424-8.

Scherer PE, Lewis RY, Volonte D, et al (1997). Cell-type and tissue-specific expression of caveolin-2. Caveolins 1 and 2 co-localize and form a stable hetero-oligomeric complex in vivo. J Biol Chem, 272, 29337-46.

Schwarz BT, Wang F, Shen L, et al (2007). LIGHT signals directly to intestinal epithelia to cause barrier dysfunction via cytoskeletal and endocytic mechanisms. Gastroenterology, 132, 2383-94.

Sendemir A, Sendemir E, Kosmehl H, et al (2008). Expression of sex hormone-binding globulin, oxytocin receptor, caveolin-1 and p21 in leiomyoma. Gynecol Endocrinol, 24, 105-12.

Shatseva T, Lee DY, Deng Z, et al (2011). MicroRNA miR-199a-3p regulates cell proliferation and survival by targeting caveolin-2. J Cell Sci, 124, 2826-36.

Shi Y, Tan SH, Ng S, et al (2015). Critical role of CAV1/ caveolin-1 in cell stress responses in human breast cancer cells via modulation of lysosomal function and autophagy. Autophagy, 11, 769-84.

Sun J, Gao J, Hu JB, et al (2012). Expression of Cav-1 in tumour cells, rather than in stromal tissue, may promote cervical squamous cell carcinoma proliferation, and correlates with high-risk HPV infection. Oncol Rep, 27, 1733-40.

Syed V, Mukherjee K, Lyons-Weiler J, et al (2005). Identification of ATF-3, caveolin-1, DLC-1, and NM23-H2 as putative antitumorigenic, progesterone-regulated genes for ovarian cancer cells by gene profiling. Oncogene, 24, 1774-87.

Tang W, Chang SB, Hemler ME (2004). Links between CD147 function, glycosylation, and caveolin-1. Mol Biol Cell, 15, 4043-50.

Tang W, Hemler ME (2004). Caveolin-1 regulates matrix metalloproteinases-1 induction and CD147/EMMPRIN cell surface clustering. J Biol Chem, 279, 11112-8.

Thompson C, Rahim S, Arnold J, et al (2017). Loss of caveolin-1 alters extracellular matrix protein expression and ductal architecture in murine mammary glands. PLoS One, 12, e0172067.

Torre LA, Bray F, Siegel RL, et al (2015). Global cancer statistics, 2012. CA Cancer J Clin, 65, 87-108.

Torres VA, Tapia JC, Rodriguez DA, et al (2007). E-cadherin is required for caveolin-1-mediated down-regulation of the inhibitor of apoptosis protein survivin via reduced beta-catenin-Tcf/Lef-dependent transcription. $\mathrm{Mol}$ Cell Biol, 27, 7703-17.

Trimmer C, Sotgia F, Whitaker-Menezes D, et al (2011). Caveolin-1 and mitochondrial SOD2 (MnSOD) function as tumor suppressors in the stromal microenvironment: a new genetically tractable model for human cancer associated fibroblasts. Cancer Biol Ther, 11, 383-94.

Wang R, He W, Li Z, et al (2014). Caveolin-1 functions as a key regulator of 17 beta-estradiol-mediated autophagy and apoptosis in BT474 breast cancer cells. Int J Mol Med, 34, 822-7.

Wiechen K, Diatchenko L, Agoulnik A, et al (2001). Caveolin-1 is down-regulated in human ovarian carcinoma and acts as a candidate tumor suppressor gene. Am J Pathol, 159, 1635-43.

Wu J, Di D, Zhao C, et al (2018). Clinical significance of Gli-1 and Caveolin-1 expression in the human small cell lung cancer. Asian Pac J Cancer Prev, 19, 401-6.

Xiong J, Wang D, Wei A, et al (2017). Deregulated expression of miR-107 inhibits metastasis of PDAC through inhibition PI3K/Akt signaling via caveolin-1 and PTEN. Exp Cell Res, 361, 316-23.

Xu J, Agyemang S, Qin Y, et al (2014). A novel pathway that links Caveolin-1 down-regulation to BRCA1 dysfunction in serous epithelial ovarian cancer cells. Enliven Chall Cancer Detect Ther, 1, 4 .

Yamaguchi H, Takeo Y, Yoshida S, et al (2009). Lipid rafts and caveolin-1 are required for invadopodia formation and extracellular matrix degradation by human breast cancer cells. Cancer Res, 69, 8594-602.

Yamasaki T, Seki N, Yoshino H, et al (2013). MicroRNA-218 inhibits cell migration and invasion in renal cell carcinoma through targeting caveolin-2 involved in focal adhesion pathway. J Urol, 190, 1059-68.

Yang H, Guan L, Li S, et al (2016). Mechanosensitive caveolin-1 activation-induced PI3K/Akt/mTOR signaling pathway promotes breast cancer motility, invadopodia formation and metastasis in vivo. Oncotarget, 7, 16227-47.

Zhang T, Hu Y, Wang T, et al (2017a). Dihydroartemisinin inhibits the viability of cervical cancer cells by upregulating caveolin 1 and mitochondrial carrier homolog 2: Involvement of $\mathrm{p} 53$ activation and $\mathrm{NAD}(\mathrm{P}) \mathrm{H}$ :quinone oxidoreductase 1 downregulation. Int J Mol Med, 40, 21-30.

Zhang T, Wang T, Cai P (2017b). Sclareol inhibits cell proliferation and sensitizes cells to the antiproliferative effect of bortezomib via upregulating the tumor suppressor caveolin-1 in cervical cancer cells. Mol Med Rep, 15, 3566-74.

Zhang W, Razani B, Altschuler Y, et al (2000). Caveolin-1 inhibits epidermal growth factor-stimulated lamellipod 
extension and cell migration in metastatic mammary adenocarcinoma cells (MTLn3). Transformation suppressor effects of adenovirus-mediated gene delivery of caveolin-1. $J$ Biol Chem, 275, 20717-25.

Zhao X, He Y, Chen H (2013). Autophagic tumor stroma: mechanisms and roles in tumor growth and progression. Int $J$ Cancer, 132, 1-8.

Zhou Y, Ren Y, Cui L, et al (2014). Differential expression of caveolin-1 in human myometrial and uterine leiomyoma smooth muscle. Am J Obstet Gynecol, 211, 496 e1- e13.

Zou W, Ma X, Hua W, et al (2015). Caveolin-1 mediates chemoresistance in cisplatin-resistant ovarian cancer cells by targeting apoptosis through the Notch-1/Akt/NF-kappaB pathway. Oncol Rep, 34, 3256-63.

Zundel W, Swiersz LM, Giaccia A (2000). Caveolin 1-mediated regulation of receptor tyrosine kinase-associated phosphatidylinositol 3-kinase activity by ceramide. $\mathrm{Mol}$ Cell Biol, 20, 1507-14.

\section{(0) $(0 \Theta$}

This work is licensed under a Creative Commons AttributionNon Commercial 4.0 International License. 